\title{
Microfluidics Assisted Fabrication of Three- Tier Hierarchical Microparticles for Constructing Bioinspired Surfaces
}

\author{
Juan Wang, ${ }^{\dagger, \ddagger}$ Hai Le-The, ${ }^{\ddagger}$ Z Zuankai Wang, ${ }^{\S}$ ( Hao Li, ${ }^{\dagger}$ Mingliang Jin, ${ }^{\dagger}$ Albert van den Berg,

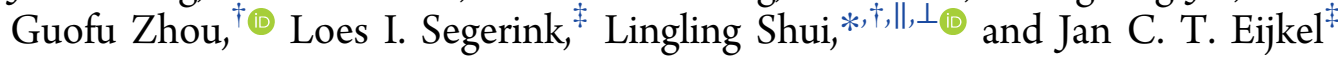

${ }^{\dagger}$ National Center for International Research on Green Optoelectronics and South China Academy of Advanced Optoelectronics, South China Normal University, Guangzhou 510006, China

${ }^{\ddagger}$ BIOS Lab-on-a-Chip Group, MESA+ Institute for Nanotechnology, Technical Medical Centre and Max Planck Center for Complex Fluid Dynamics, University of Twente, Enschede 7522NB, The Netherlands

${ }^{\S}$ Department of Mechanical and Biomedical Engineering, City University of Hong Kong, Hong Kong 999077, China

"School of Information and Optoelectronic Science and Engineering, South China Normal University, Guangzhou 510006, China

Supporting Information

\begin{abstract}
Construction of textured bioinspired surfaces with refined structures that exhibit superior wetting properties is of great importance for many applications ranging from self-cleaning, antibiofouling, anti-icing, oil/water separation, smart membrane, and microfluidic devices. Previously, the preparation of artificial surfaces generally relies on the combination of different approaches together, which is a lack of flexibility to control over the individual architecture unit, the surface topology, as well as the complex procedure needed. In this work, we report a method for rapid fabrication of three-tier hierarchical microunits (structures consisting of multiple levels) using a facile droplet microfluidics approach.

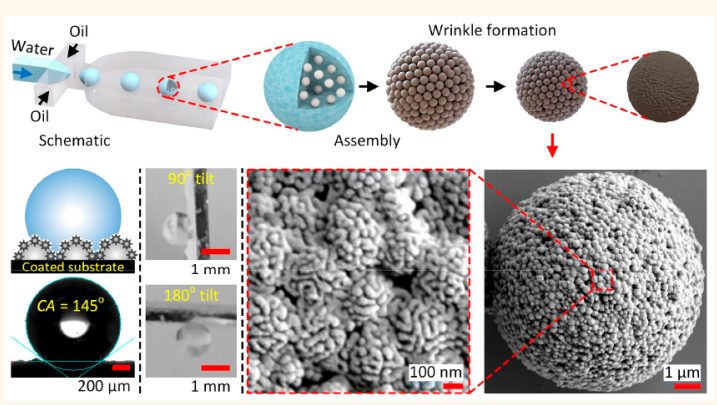
These units include the first-tier microspheres consisting of the second-tier close-packed polystyrene (PS) nanoparticles decorated with the third-tier elegant polymer nanowrinkles. These nanowrinkles on the PS nanoparticles are formed according to the interfacial instability induced by gradient photopolymerization of $N$-isopropylacrylamide (NIPAM) monomers. The formation process and topologies of nanowrinkles can be regulated by the photopolymerization process and the fraction of carboxylic groups on the PS nanoparticle surface. Such a hierarchical microsphere mimics individual units of bioinspired surfaces. Therefore, the surfaces from self-assembly of these fabricated two-tier and three-tier hierarchical microunits collectively exhibit "gecko" and "rose petal" wetting states, with the micro- and nanoscale structures amplifying the initial hydrophobicity but still being highly adhesive to water. This approach offers promising advantages of high-yield fabrication, precise control over the size and component of the microspheres, and integration of microfluidic droplet generation, colloidal nanoparticle self-assembly, and interfacial polymerization-induced nanowrinkles in a straightforward manner.
\end{abstract}

KEYWORDS: three-tier, hierarchical structure, microfluidics, nanowrinkle, bioinspired surface

W rinkled surfaces in nature have inspired scientists to mimic them with artificial topological surfaces with high spatial periodicity. Such structures on the micro- and nanoscale show optical, biological, electronic, or acoustic properties due to their wave interference phenomena, exceptional hydrodynamic properties, enhanced adhesion force, low surface energy, and/or large specific surface area. ${ }^{1,2}$ The integration of wrinkled surfaces with multiple hierarchical (i.e., structured on multiple scales in one unit) architectures even further improves the performance in catalytic support, ${ }^{3}$ electrodes, ${ }^{4}$ controllable superwetting modulation, ${ }^{5-7}$ digital microfluidics, ${ }^{8}$ microfluidic sieves, ${ }^{9}$ surface enhanced Raman spectroscopy (SERS), ${ }^{10,11}$ tracking and identification technology, ${ }^{12,13}$ flexible electronics, ${ }^{14-16}$ diffraction gratings, ${ }^{17}$ separation science, ${ }^{18}$ drug delivery, ${ }^{19}$ tissue engineering, ${ }^{20,21}$ industrial field-like abrasives material, ${ }^{22}$ and environment protection materials. $^{23}$ Therefore, study and construction of the unit of such materials/surfaces become prominent to understand the relationship between structures and functionalities.

Received: January 10, 2019

Accepted: March 11, 2019

Published: March 11, 2019 

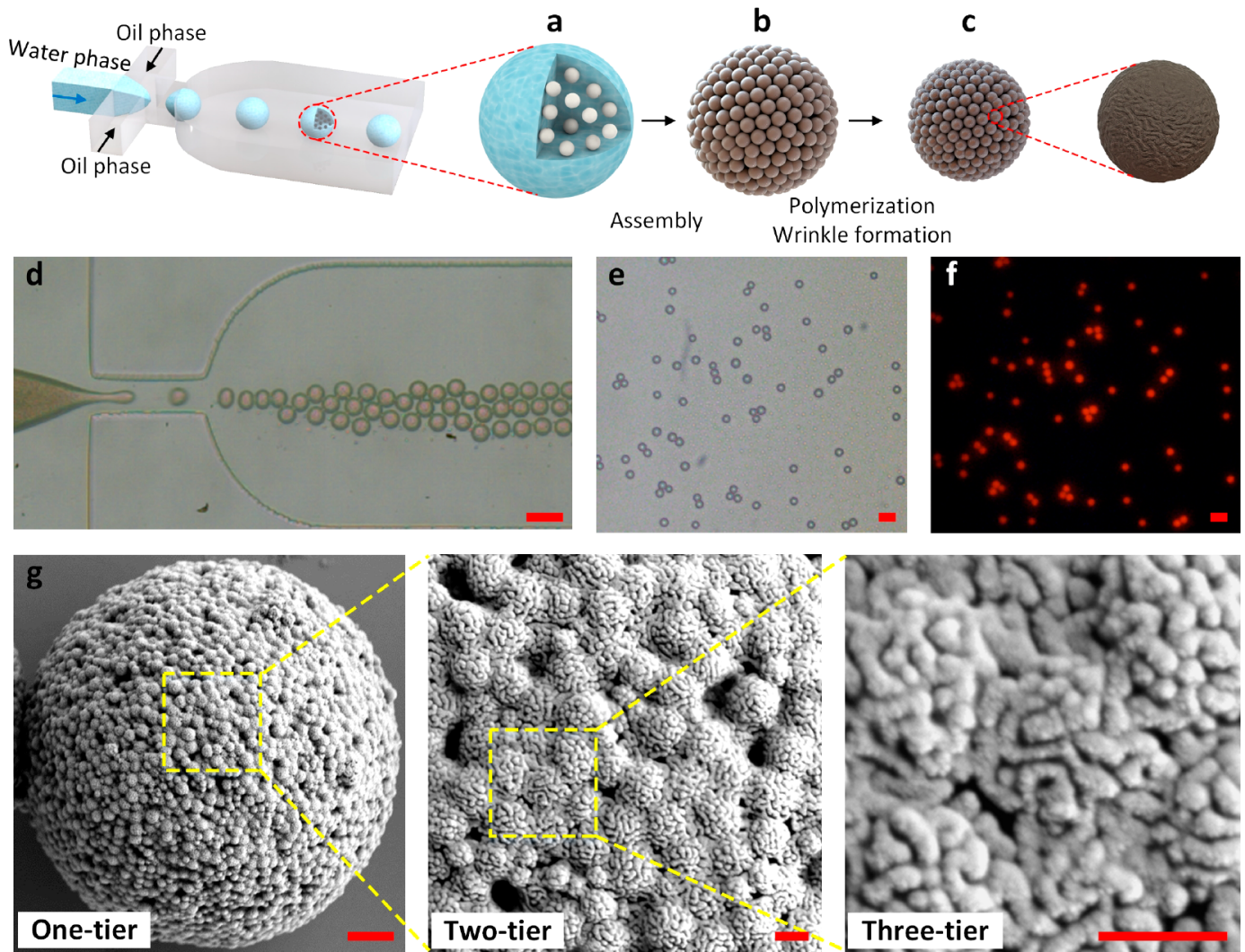

Figure 1. Schematic diagram of the preparation of three-tier hierarchical microspheres using a droplet-based microfluidic device. (a) Generation of emulsion droplets. (b) Assembly of nanoparticles confined in the droplet. (c) Photopolymerization of the droplet and the formation of a solid hierarchical microsphere with nanowrinkles. (d) Optical microscopic image of the droplet generation in a flow-focusing microfluidic device. (e) Optical microscopic image of collected droplets under bright field mode and (f) the corresponding fluorescent field observation excited by a light source of $365 \mathrm{~nm}$ wavelength. Scale bars in $(\mathrm{a}-\mathrm{f})$ represent $50 \mu \mathrm{m}$. (g) HR-SEM (scale bar: $1 \mu \mathrm{m})$ and close-up images (scale bar: $200 \mathrm{~nm}$ ) of a $f$-PS@MS at the microsphere, nanoparticle, and nanowrinkle levels from left to right.

A summary of various methods for fabrication of artificial hierarchical structures on two-dimensional (2D) polymer films with wrinkled surfaces has been reported by Chen and Yang. ${ }^{24}$ The underlying mechanism of the wrinkle formation on the film is attributed to the applied stress on their surface. When this stress is larger than a critical value, it leads to an interfacial instability due to the mismatch of mechanical properties like the Young's modulus in the equilibrium state between different layers-normally between a stiff skin layer and soft substrate. The applied stress is commonly raised by mechanical (e.g., stretching, compressive force), ${ }^{9,25,26}$ thermal (e.g., electron irradiation), ${ }^{27,28}$ physical (e.g., osmotically driven), ${ }^{29}$ and chemical (e.g., solvent swelling, ultraviolet (UV) irradiation, oxygen plasma treatment) approaches. $^{26,30}$

Recently, hierarchical architectures have been developed for three-dimensional (3D) materials and have attracted intense attention. The functional properties (defined as stimuliresponsive) of $3 \mathrm{D}$ hierarchical structures are determined by their chemical composition and tailored surface topology, exhibiting the advantages of high spatial frequency, enlarged specific surface roughness and area. In particular, controlling of surface topology enables the control of interfacial phenomena such as fluid wetting, flow, and adhesion. ${ }^{31,32}$ These hierarchical structures have enhanced functionalities by their surface topology on the micro- and nanoscopic length scale, thus broadening their applications in many fields. For example, regarding the application of wetting materials, tailoring surface roughness can give rise to an amplified hydrophilicity/ hydrophobicity for the film surfaces. Accordingly, an effective approach for fabricating superwetting surfaces is to create hierarchical structures. ${ }^{33,34}$ Hierarchical bioinspired structures that are, for example, the mimicry of gecko feet, a mosquito eye, and a rose petal have been fabricated through either the topdown methods of lithography ${ }^{35,36}$ and laser writing ${ }^{37}$ or through bottom-up approaches like self-assembly of colloidal particles. $^{36,38}$

Artificially wrinkled surfaces on particles are typically constructed in two separate steps, that is, synthesis of the polymer template and postprocessing. The polymer templates can be synthesized using flow lithography, ${ }^{39}$ electro-spraying, ${ }^{40}$ or microfluidic techniques. ${ }^{41-43}$ Subsequently, the exposed surfaces are modified by using additional material coating, ${ }^{44}$ plasma etching, ${ }^{45}$ surface chemical oxidation, ${ }^{46}$ chemical modification, ${ }^{47}$ solvent evaporation, ${ }^{3,48}$ electron irradiation, ${ }^{27}$ or UV irradiation. ${ }^{49}$ Although effective, these fabrication methods are time-consuming and require complex processes. It is worth mentioning that among these technologies, the droplet-based microfluidic technique stands out by its production of droplet templates with high uniformity in size and tunability in particle geometry and chemical composition. ${ }^{42,50}$ Moreover, this method can be easily integrated with other approaches (as a hybrid approach: combination of topdown and bottom-up method), thus enabling continuous construction of 3D hierarchical microparticles with different chemical and physical properties. Liu et al. reported a dropletbased microfluidic method for fabricating polymer micro- 



Figure 2. (a) AFM image of the surface of a $f$-PS@MS and (b) the corresponding dimensions measured along the red dashed line. (c) HR-SEM (scale bar: $1 \mu \mathrm{m}$ ) and a close-up image (scale bar: $200 \mathrm{~nm}$ ) of the cross-sectional morphology of a fabricated $f$-PS@MS cut by using a focus ion beam.

particles. $^{3}$ The surface textures on polymer microparticles could be tuned via interfacial instability triggered by emulsion droplets shrinking. Metal nanoparticle-loaded polymer composite particles have also been synthesized via microfluidic approach integrated with in situ photopolymerization and photoreduction. ${ }^{11}$ These composite particles could serve as sensing units for molecular detection. However, these microparticles only show two-tier hierarchy of nanostructures on the microparticles.

In this work, we report a hybrid approach for rapid construction of three-tier hierarchical microspheres, using a droplet-based microfluidic technique to produce microparticles with patterned nanoparticles (via colloid nanoparticle selfassembly) covered with a convoluted (wrinkled) surface (via gradient photopolymerization). The fabricated three-tier microspheres can be applied as uniform units to form bioinspired surfaces, showing controllable surface wettability. This method has the advantages of producing microspheres with high uniformity in physical size and flexibility in material selection. Using this integrated platform, the entire three-tier structure is synthesized: the polymerization reaction for producing nanowrinkles at tens of nanometer scale (the third tier), the colloidal self-assembly to form close-packed nanopatterns at the hundreds of nanometer scale (the second tier), and the droplet-based microfluidics for constructing particles at the tens to hundreds of micrometer scale (the first tier). The distribution of nanowrinkles and nanoparticles on the microsphere surface is of critical significance for structure-dominant applications. In this way, both the microspheres and the assembled surfaces can be tuned chemically and physically. Such hierarchical microspheres show similar surface structures to the unit of bioinspired surfaces. As a result, the surfaces made from the assembled two- and three-tier microspheres demonstrate the "gecko" and "rose petal" wetting states, respectively.

\section{RESULTS AND DISCUSSION}

Fabrication of Three-Tier Hierarchical Units of Microspheres Consisting of Nanoparticles Covered with Nanowrinkles. Three-tier hierarchical microspheres with nanowrinkles were successfully fabricated using droplet microfluidics. A microfluidic device with a flow-focusing geometry was used to generate water-in-oil emulsion droplets with high uniformity (coefficient of variation of $1.5 \%$ ) and high yield (>1000 microspheres per second). Herein, a suspension containing $3 \mathrm{w} / \mathrm{v} \%$ carboxylic-functionalized polystyrene $(f$ PS) nanoparticles, $5 \mathrm{wt} \%$ NIPAM as curable monomer, and 0.5 wt \% N,N-methylene bis (acrylamide) (MBA) as cross-linker was used as the water phase (droplet). The oil (continuous) phase was $n$-hexadecane with $19 \mathrm{wt} \%$ sorbitane monooleate sorbitan oleate (Span80) as surfactant to stabilize the produced droplets and 5 wt \% 2,2-diethoxyacetophenone (DEAP) as a photoinitiator to initiate the photopolymerization reaction. The concentrations of NIPAM and MBA in the water phase and Span 80 and photoinitiator in the oil phase were kept constant in all experiments. The water phase was sheared off to form monodispersed emulsion droplets by the oil phase at the flowfocusing area. Generated droplets were collected in a beaker containing oil phase, as shown in Figure 1a. As time evolved, $f$-PS nanoparticles confined in the emulsion droplet organized and 

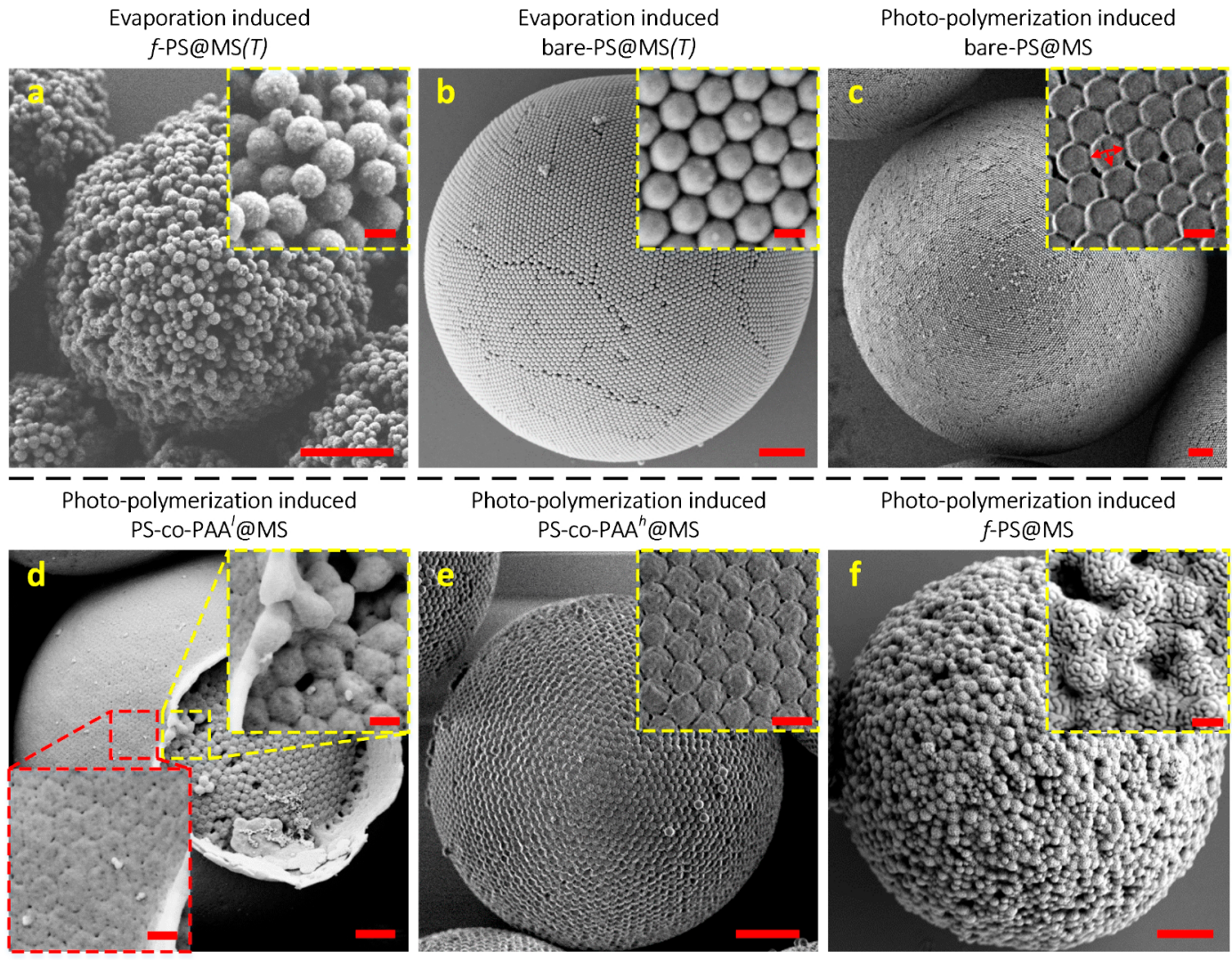

Figure 3. Effects of water evaporation and polymerization on the wrinkle formation. HR-SEM images of (a)f-PS@MS(T), (b) bare-PS@MS(T), (c) bare-PS@MS, (d) PS-co-PAA $@ M S$, (e) PS-co-PAA $@ M S$, and (f) $f$-PS@MS with the corresponding close-up images. The scale bars are 2 $\mu \mathrm{m}$ and $200 \mathrm{~nm}$ in the main and inset images, respectively. Note that there was no deposition of metal films prior to the HR-SEM characterization.

assembled, driven by interfacial tension and electrostatic repulsion, ${ }^{51}$ producing close-packed nanopatterns on the droplet surface (Figure 1b). To obtain the well-ordered nanopatterns, the collected droplets in the beaker containing the oil phase were kept for more than $1 \mathrm{~h}$. Afterward, these droplets were photopolymerized through UV-induced interfacial free-radical polymerization (IFRP), yielding solid microspheres (Figure 1c). The volume shrinkage of produced liquid microdroplets into solid microspheres was approximately 96.38\% (see the calculation in Supporting Information). These solid microspheres showed three-tier hierarchical structure, including the first tier of microframe, the second tier of close-packed nanopatterns assembled from $f$-PS nanoparticles, and the third tier of nanowrinkles on every individual $f$-PS nanoparticle surface caused by the gradient-UV photopolymerization. Figure 1d shows an optical microscope (OM) image of droplet generation in the microfluidic device and the collected monodispersed droplets observed under the bright field (Figure 1e) and fluorescent field (Figure 1f) modes. The fluorescent image shows bright red color under $365 \mathrm{~nm}$ excitation due to the imbedded quantum dots $(\mathrm{CdSe} / \mathrm{ZnS})$ in the center of purchased $f$-PS nanoparticles. High-resolution scanning electron microscope (HR-SEM) images show the three-tier structures of the polymerized microspheres (Figure $1 \mathrm{~g})$.

Figure 2 shows the surface of a three-tier hierarchical microsphere (f-PS@MS) and the corresponding dimensions of the nanowrinkles measured by an atomic force microscope (AFM). From the observation, the nanowrinkles are in the sub-
$30 \mathrm{~nm}$ range, and their distribution on every individual nanoparticle is relatively uniform. To further view the inside of the microspheres, we cut a f-PS@MS using a focused ion beam. Figure $2 \mathrm{c}$ shows the cross section of the cut surface. It reveals that the microsphere was fully filled with $f$-PS nanoparticles and nanowrinkles exist on each $f$-PS nanoparticle. The flat surface of the cut area in the HR-SEM image, hereby, was caused by heat melting during the focus ion beam cutting.

The produced f-PS@MS exhibits a large surface-to-volume ratio of $\sim 3 \times 10^{7} \mathrm{~m}^{-1}$ (about 47 times larger than that of a smooth microsphere at the same diameter), high spatial frequency of $\sim 3.3 \times 10^{5} \mathrm{~mm}^{-1}$ (which is defined as the frequency of the nanowrinkle structure repetition per unit of distance), and high-density nanogaps of $\sim 422$ nanogaps per $\mu \mathrm{m}^{2}$ (a gap between two adjacent peaks of the nanowrinkles is defined as a nanogap) (Figures S1-S2). The surface-to-volume ratio was calculated by multiplying the measured surface area of individual nanoparticles by the number of particles inside a microsphere. The surface area of a nanoparticle with nanowrinkles was calculated based on the AFM measured average nanowrinkle dimensions and gaps. It is therefore worth mentioning that these values might be slightly overestimated. Therefore, these f-PS@MS are expected to have high potential for coating materials (surface wettability control via micro- and nanoscale structures), molecular detection (localized surface plasmon resonance due to the presence of nanogaps), bioassays (high adhesive surface), and catalysis support (large specific surface area). 


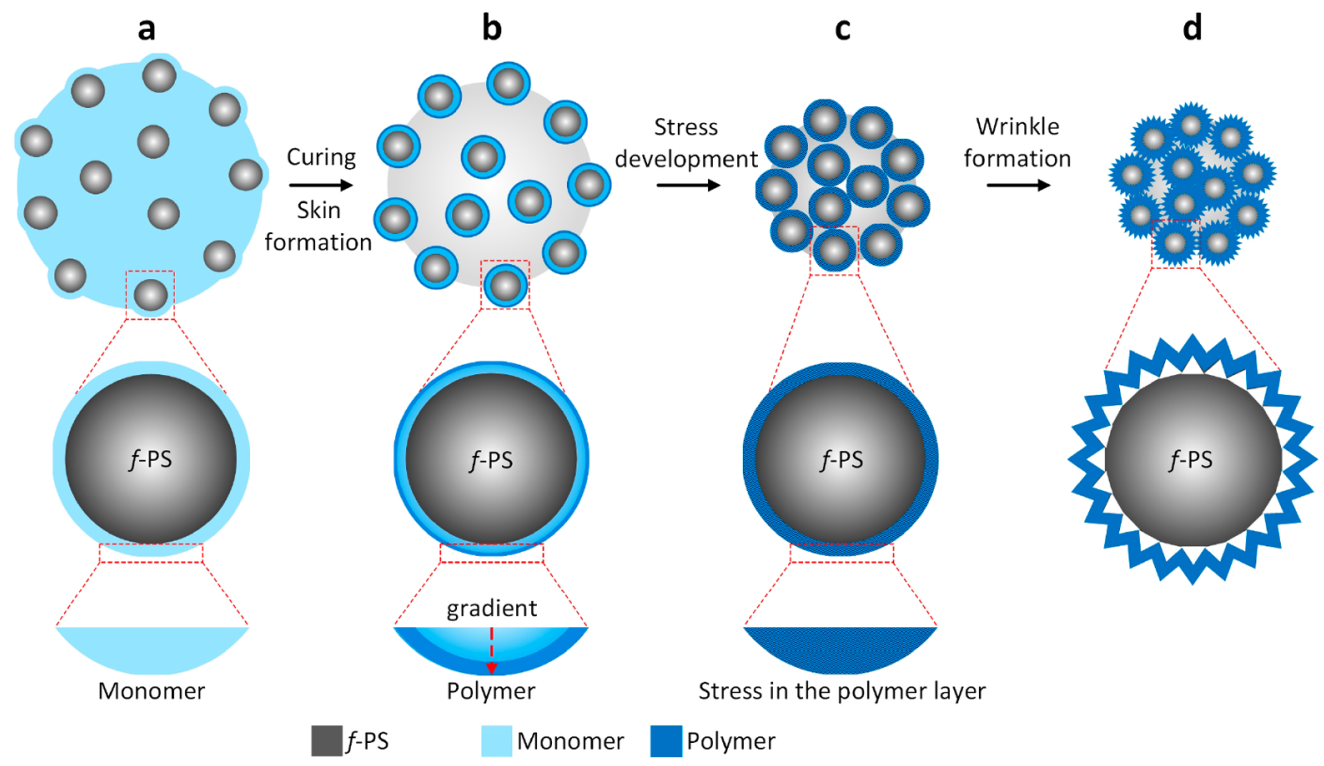

Figure 4. Schematic diagram of the formation of wrinkled surfaces on a $f$-PS@MS. (a) Droplet with close-packed $f$-PS nanoparticles before curing. (b) Initial state of droplet curing and formation of a thin stiff layer at the top surface. (c) Further gradient-curing (cross-linking reaction) and stress development. (d) Wrinkle formation on the $f$-PS nanoparticle surface.

Mechanism of Nanowrinkle Formation. As presented in previous literatures, ${ }^{3,18}$ the formation of nanowrinkles is commonly due to the volume shrinkage resulting from solvent evaporation and/or polymerization reactions occurring on the structure surface. To understand the mechanism of nanowrinkle formation in this work, a series of comparative experiments were designed and conducted.

In the first comparative experiment, a suspension of pure $f$-PS nanoparticles was used as the water phase to produce the droplets, and solid microspheres were subsequently produced via solvent evaporation at $60{ }^{\circ} \mathrm{C}$ overnight. These particles were termed as f-PS@MS $(T)$. The morphology of these f-PS@ $\operatorname{MS}(T)$ is shown in Figure 3 a. As can be seen, all $f$-PS nanoparticles assembled into micrometer clusters without wrinkle formation on their surface, compared with that in Figure 1g. The surface morphology of single $f$-PS nanoparticle was found to be similar to the virgin one (Figure S 3f). This indicates that the volume shrinkage during the water evaporation process from liquid droplets to solid microspheres did not induce wrinkle formation on the $f$-PS nanoparticle surface.

Moreover, a pure suspension of bare-PS nanoparticles (bare means without carboxylic acid modification) was used as the water phase to generate the droplets and subsequently evaporated in an oven at $60{ }^{\circ} \mathrm{C}$ overnight, forming into solid microspheres with well-ordered and close-packed nanopatterns, termed as bare-PS@MS(T), as presented in Figure 3b. Due to the difference in the uniformity and stability between $f$-PS $(\zeta$ potential: $-22 \mathrm{mV}$ ) and bare-PS ( $\zeta$ potential: $-58 \mathrm{mV}$ ) nanoparticles, the produced microspheres show different nanopatterns. It is worth mentioning that the bare-PS nanoparticles were synthesized by anionic polymerization with the assistance of sodium dodecylbenzenesulfonate as the emulsifier and surfactant to improve their stability. However, there was also no wrinkle formation on the nanoparticle surface (Figure 3b).

Furthermore, to determine the occurrence of chemical reactions between the nanoparticles and the NIPAM monomers, a mixture of bare-PS nanoparticles with the same concentration of NIPAM and MBA was used as the water phase to generate the droplets, which were subsequently photopolymerized by UV irradiation to form microspheres termed as bare-PS@MS. Figure $3 c$ shows the top-view HR-SEM images of a fabricated bare-PS@MS, exhibiting a smooth surface on each bare-PS nanoparticle. It was found that a NIPAM polymer layer filled in the space between the bare-PS nanoparticles (see red arrows in Figure 3c), indicating the occurrence of NIPAM cross-linking reaction. In contrast to f-PS@MS (Figure 1g), the bare-PS@MS (Figure 3c) shows a smooth surface, suggesting a critical role of the surface carboxylic groups on the surface of the PS nanoparticles on the formation of nanowrinkles during photopolymerization-induced solidification from droplets to particles.

To further investigate the effect of the surface carboxylic groups on the photopolymerization process, copolymer polystyrene nanoparticles containing different molar fractions of carboxylic groups were employed to fabricate the microspheres. In four separate experiments, bare-PS (no carboxylic groups), PS-co-PAA ${ }^{l}$ (containing $10 \%$ carboxylic groups in molar fraction), PS-co-PAA ${ }^{h}$ (containing $18.5 \%$ carboxylic groups in molar fraction), and f-PS nanoparticles (fully functionalized with carboxylic groups on the surface) were mixed with 5 wt $\%$ NIPAM and 0.5 wt $\%$ MBA, as the aqueous phases to prepare hierarchical microspheres denoted as barePS@MS, PS-co-PAA $@ M S$, PS-co-PAA ${ }^{h} @ M S$, and f-PS@MS, respectively. The collected microdroplets were suspended in the continuous phase of $n$-hexadecane containing 19 wt $\%$ Span 80 and $5 \mathrm{wt} \%$ DEAP, followed by polymerization using UV irradiation. Figure $3 c-f$ shows the HR-SEM images of these fabricated bare-PS@MS, PS-co-PAA $@ M S, P S-c o-P A A^{h} @ M S$, and $f$-PS@MS. Without carboxylic groups on surface, the barePS nanoparticles could assemble to nice and smooth microspheres via photopolymerization (Figure $3 \mathrm{c}$ ). There was no obvious difference found on both the microspheres and the nanoparticle surfaces. On the surface of PS-co-PAA $@$ MS in Figure 3d (also Figure S4a), a layer of NIPAM polymer was observed with imprinted traces of the encapsulated nanoparticles. Under this NIPAM polymer layer, PS nanoparticles were closely packed with rough surfaces, indicating that the 
Bare-PS@MS

a

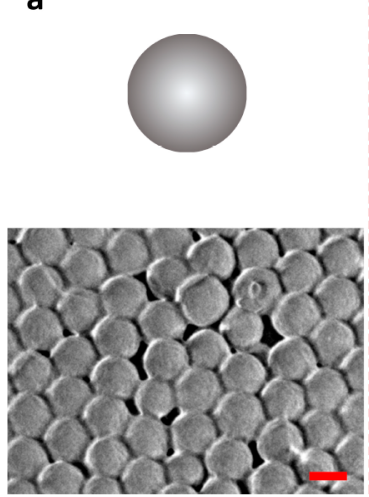

PS-co-PAA'@MS
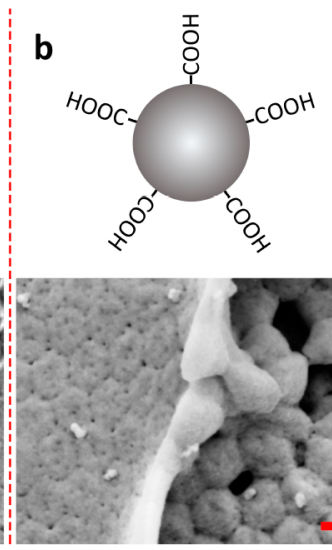

PS-Co-PAA ${ }^{h} @ M S$

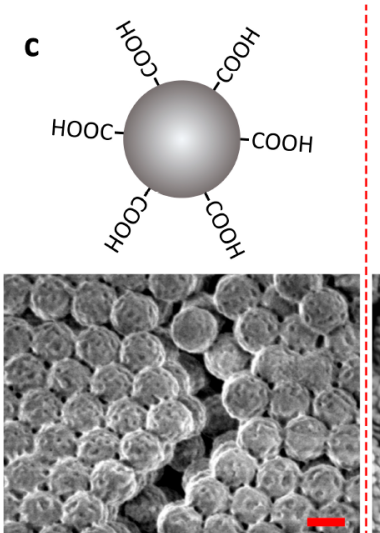

f-PS@MS

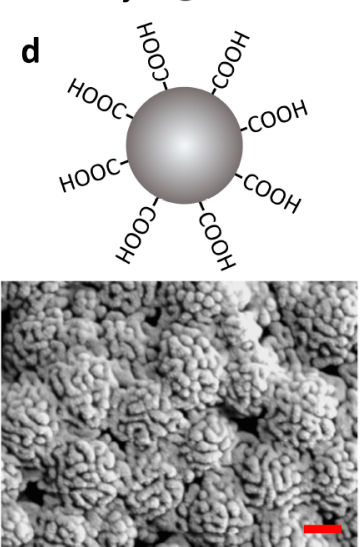

Figure 5. Schematic and corresponding experimental results (HR-SEM images) of the produced hierarchical microsphere surfaces using different PS nanoparticles: (a) bare-PS, (b) PS-co-PAA ${ }^{l}$, (c) PS-co-PAA ${ }^{h}$, and (d)f-PS. Scale bars represent $200 \mathrm{~nm}$.

cross-linked NIPAM monomers remained attached to the surface of PS-co-PAA $@$ MS. Moreover, on the PS-co-PAA ${ }^{h} @ M S$ surface in Figure 3e (also Figure S4b), close-packed and wellordered nanoparticles were observed with obvious nanowrinkled surface. On the f-PS@MS surface, nanowrinkles were found fully covering the nanoparticle and microsphere surfaces, as shown in Figures $3 \mathrm{f}$ and $2 \mathrm{c}$. These results suggest that, during the photopolymerization process, the microsphere and nanoparticle surfaces change obviously with the fraction of carboxylic groups of the PS nanoparticles.

By comparing these microspheres constructed by evaporation-induced solidification composed of $f$-PS (Figure 3a) and bare-PS (Figure $3 \mathrm{~b}$ ) nanoparticles and photopolymerizationinduced solidification composed of bare-PS (Figure 3c), PS-coPAA $^{l}$ (Figure 3d), PS-co-PAA ${ }^{h}$ (Figure $3 \mathrm{e}$ ), and $f$-PS (Figure $3 \mathrm{f}$ ) nanoparticles, we can conclude that both the photopolymerization process and the fraction of carboxylic groups on PS nanoparticle surface play critical roles for the nanowrinkle formation.

Therefore, we propose the following mechanism to explain the wrinkle formation. Chemically, the NIPAM molecule contains an amine group. When there is no carboxylic group on the PS nanoparticle surface as in the case of the bare-PS nanoparticles, the NIPAM monomers diffuse freely in the water droplets and cross-link upon UV irradiation to form a continuous network, filling the space among the nanoparticles. NIPAM and PS behave as two separate phases, and NIPAM polymer does not bind to the PS nanoparticle surface. Additionally, there was no attractive electrostatic force between the NIPAM monomers and the bare-PS nanoparticles, as the NIPAM monomers are neutrally charged. Therefore, the NIPAM monomers do not prefer to attach on the surface of the bare-PS nanoparticles, leading to no nanowrinkle formation. However, when the PS nanoparticles are functionalized with carboxylic groups such as $f$-PS, hydrogen bonds can be formed between the carboxylic groups on the PS nanoparticle surface and the amine groups from NIPAM. ${ }^{52,53}$ The formation of nanowrinkles on each $f$-PS nanoparticle results from the photogradient polymerization upon UV irradiation. ${ }^{54}$

Figure 4 schematizes the wrinkle development procedure. The droplet, containing NIPAM as curable monomer and watersoluble MBA as cross-linker, is UV cured via interfacial freeradical polymerization. ${ }^{55}$ Before UV irradiation, the water phase (with NIPAM and MBA) is confined to the individual microdroplets, filling the nanogaps between the nanoparticles (Figure 4a). Upon UV irradiation, the photoinitiator molecules in the oil phase become radicals and diffuse into the water-oil interface to activate NIPAM monomers to start the cross-linking reaction. Consequently, the NIPAM monomers and produced oligomers bind to the surface of carboxylic-functionalized PS nanoparticles due to the hydrogen bonds (Figure $4 \mathrm{~b}$ ). When the NIPAM monomers and oligomers bind to the nanoparticle surface, polymer remains attached to the nanoparticle surface, which cannot move freely. It is expected that the top layer close to the continuous oil phase is first polymerized to become a skin layer, which is harder than the inner layer close to the nanoparticle due to the gradient in cross-linking reaction. Additionally, NIPAM polymer is known to be highly sensitive to temperature changes. ${ }^{56}$ In our UV curing system, the chamber temperature was approximately $65^{\circ} \mathrm{C}$ during the exposure. Such temperature is higher than its low critical solution temperature (LCST of NIPAM polymer) of $32{ }^{\circ} \mathrm{C}$, causing the NIPAM polymer to become hydrophobic, expelling water. ${ }^{57}$ This leads to the drastic shrinkage of the formed skin layer. When the polymer is strongly attached to the nanoparticle surface, no detachment takes place, and the shrinkage of NIPAM polymer produces an in-plane tensile stress in the skin layer. The elastic modulus of the NIPAM polymer layer as a skin layer increases by an order of magnitude compared to that of the inner soft layer. ${ }^{57}$ This difference in the elastic modulus between the two layers essentially results in the formation of nanowrinkles. On the other hand, due to the gradient in the chemical potential during the cross-linking reaction, the monomers/oligomers tend to diffuse toward the hard skin layer, thus resulting in a swelling tendency. Satisfaction of further cross-linking promotes monomers/ oligomers to diffuse deeper into the skin, which as a result slowly changes the state of stress of the skin from tensile to compressive. Therefore, a competition between the tensile stress (caused by the shrinkage of the skin layer from chemical reaction) and the compressive stress (resulted from the swelling) is built (Figure 4c). In order to release the stresses, an out-of-plane deformation accompanied by nanowrinkles occurs (Figure 4d).

On a flat surface, the wavelength of nanowrinkles can be characterized by the equation of $\lambda \approx 2 \pi h\left(E_{\mathrm{S}} / E_{\mathrm{B}}\right)^{1 / 3}$, where $h$ is the thickness of the skin layer, $E_{\mathrm{S}}$ and $E_{\mathrm{B}}$ are the Young's modulus of the skin hard and inner soft layers, respectively. ${ }^{58,59}$ This indicates that the characteristics of the nanowrinkles can be 


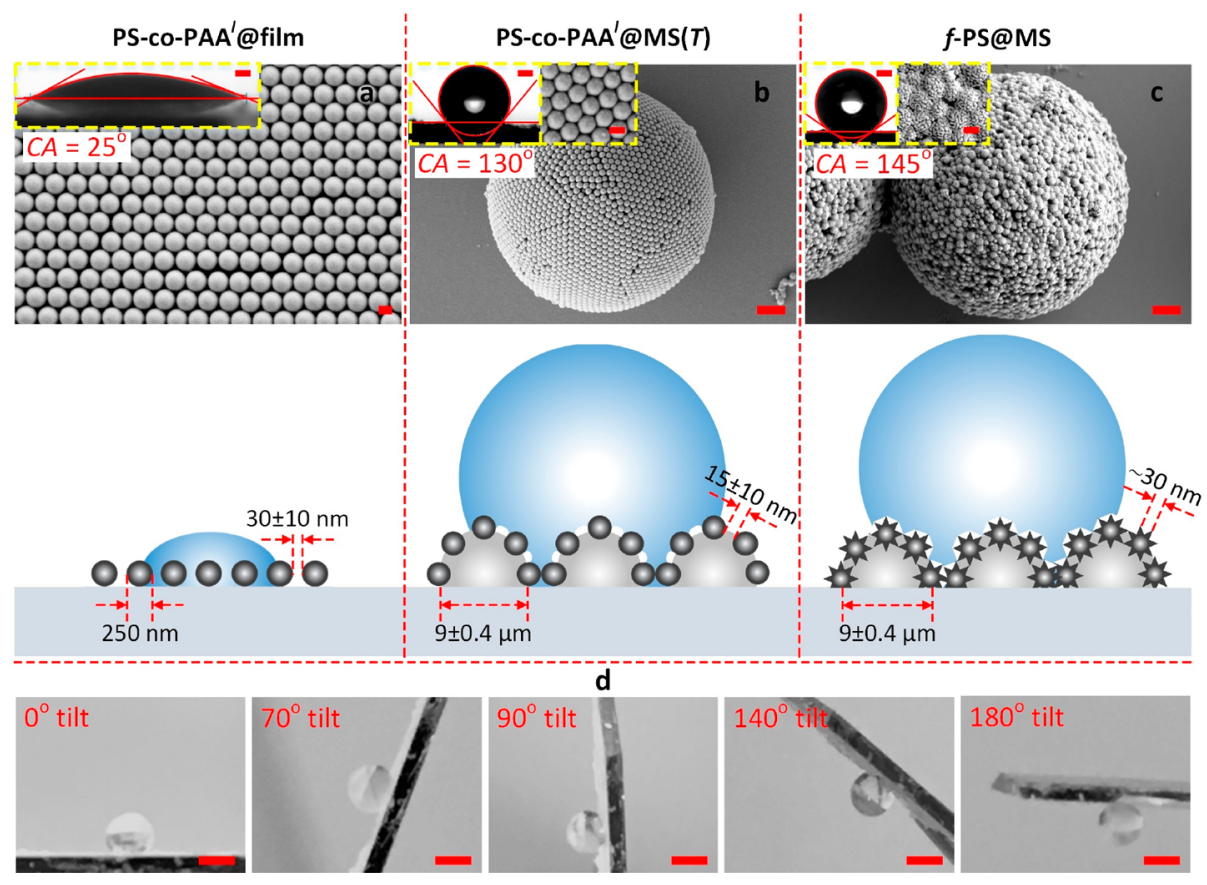

Figure 6. HR-SEM images of films prepared by assembly of (a) PS-co-PAA nanoparticles (scale bar: 200 nm), (b) PS-co-PAA @MS(T) (scale bar: $1 \mu \mathrm{m}$ ), and (c) $f$-PS@MS (scale bar: $1 \mu \mathrm{m})$. Inset HR-SEM images in $(\mathrm{b})$ and $(\mathrm{c})$ show the amplified nanopattern surfaces (scale bar: 200 $\mathrm{nm}$ ). Inset optical images in $(\mathrm{a}-\mathrm{c})$ show the $C A$ measurements of $2 \mu \mathrm{L}$ water drops sitting on the corresponding films (scale bars: $200 \mu \mathrm{m})$. The schematic diagrams on the bottom rows show corresponding geometries and parameters of the three films. (d) A water drop standing on a film covered by the three tier $f$-PS@MSs, tilted at different angles (scale bars: $1 \mathrm{~mm}$ ).

tailored by the physical properties of the skin hard layer, the inner soft layer, and the thickness of the skin layer. When the temperature is above $40{ }^{\circ} \mathrm{C}$, the modulus of the skin layer is found to be approximately $600 \mathrm{kPa}$, whereas the modulus of the soft layer is about $85 \mathrm{kPa}{ }^{57}$ Using the mentioned equation with the measured wavelength of the nanowrinkles using AFM, the thickness of the skin layer is estimated to be approximately $3 \mathrm{~nm}$. It has to be pointed out that the modulus of NIPAM polymer is expected to be slightly different from that of the nanogels reported by Schmidt et al. ${ }^{57}$ since the formed NIPAM polymer layer is 1 order of magnitude thinner than the nanogels, which can lead to a difference in the cross-linking density.

We conclude from the above investigation that the surface topology of hierarchical microspheres can be precisely controlled by changing the fraction of carboxylic groups on the PS nanoparticle surface, as shown in Figure 5. With a high density of carboxylic groups on the PS nanoparticle surface, the produced wrinkles show high spatial frequency and roughness.

Bioinspired "Gecko-like" and "Rose Petal-like" Surfaces from Self-Assembly of Two- and Three-Tier Microspheres. Surfaces making use of versatile topological structures have attracted a lot of attention due to their wide applicability in waterproofing, self-cleaning, antifouling, and anti-icing. ${ }^{34,60-62}$ Surface wettability is strongly dependent on both the tailored structure and chemical modification, thereby tuning the adhesion and friction forces between fluids and solid surfaces. We investigated the effect of the surface topography on the surface wettability of different films composed of closely packed PS nanoparticles (PS-co-PAA ${ }^{l}$ ), two-tier hierarchical microspheres constructed by PS nanoparticles(PS-co-PAA $@ M S(T)$ ), or three-tier hierarchical microspheres constructed by PS nanoparticles covered with nanowrinkles ( $f$-PS@MS). In Figure 6a, a film of closely packed PS nanoparticles was assembled by using PS-co-PAA ${ }^{l}$ nanoparticles $(250 \mathrm{~nm}$ in diameter). In Figure
$6 \mathrm{~b}$ (see also AFM images in Figure S5), the two-tier hierarchical microspheres (PS-co-PAA ${ }^{l} @ M S(T)$ ) were generated from the same PS-co-PAA ${ }^{l}$ nanoparticles using the droplet-based microfluidics approach with evaporation-induced assembly and solidification. In Figure 6c (see also AFM images in Figure 2), the three-tier hierarchical microspheres (f-PS@MS) were produced from $f$-PS nanoparticles of $250 \mathrm{~nm}$ in diameter using the same method with photopolymerization-induced solidification. The sub-30 nm nanowrinkles on f-PS@MS led to significant enhancement of surface hydrophobicity, suggested by the increase in water contact angle ( $C A)$ measurements.

Physical equations have been used to express the relationship between the surface roughness and wettability, typically the Wenzel and Cassie-Baxter equations. In the Wenzel state, the water drop totally wets the substrate, forming a continuous three-phase contact line (TCL), resulting in a high adhesive force (high CA hysteresis). ${ }^{63}$ In contrast, in the Cassie-Baxter state, ${ }^{64}$ air is trapped at the interface between the water drop and the substrate, forming a nonwetting contact mode and a discontinuous and unstable TCL, resulting in low adhesion (low $C A$ hysteresis). The Wenzel and Cassie-Baxter states are considered as ideal models for a drop sitting on a superhydrophobic surface. However, practically, there are several intermediate states such as the "gecko" 65 and the "rose petal" states, ${ }^{66}$ existing on both natural and artificial hierarchical structures. In the "gecko" state, a water drop is sucked into the top part of nanoscale capillaries, resulting in sealed air pockets trapped in the bottom of the nanoscale spaces. This leads to the formation of a negative pressure and thus produces a high adhesive force. ${ }^{65}$ In the "rose petal" state, a water drop only partially wets the textured structure of a superhydrophobic surface. When a water drop sits on a "rose petal" surface, the water impregnates only into the large-scale micropapillae of the petal without filling the nanofolds on top of the micropapillae, 
thus leaving air trapped at the nanofolds. Such a wetting state enables the water drop to be tightly adhered to the rose petal surface, even when it is turned upside-down. ${ }^{66}$

The films prepared from PS-co-PAA ${ }^{l}$ nanoparticles, PS-coPAA $@$ MS $(T)$, and $f$-PS@MS showed CAs of $25^{\circ}, 130^{\circ}, 145^{\circ}$ in Figure $6 \mathrm{a}-\mathrm{c}$, respectively. The surface of assembled PS-co-PAA ${ }^{l}$ nanoparticles showed a Wenzel state with the water drop wetting the surface and forming a continuous TCL, as shown in Figure 6a. This 2D film was featured with high-density nanogaps with $30 \pm 10 \mathrm{~nm}$ distance among neighboring PS-co-PAA ${ }^{l}$ nanoparticles. Such a structure leads to much more hydrophilic property in comparison with a flat polystyrene film ( $C A$ of $\left.\sim 90^{\circ}\right)$. $^{66}$

Figure $6 \mathrm{~b}$ shows a surface composed of the well-patterned two-tier microspheres of PS-co-PAA $@$ MS $(T)$, including the micropatterns from assembled microspheres as the frames (analogy to the setae of gecko's foot) and the nanopatterns of closely packed nanoparticles (analogy to the spatulae on setae), which shows a "gecko" state. ${ }^{65}$ From the AFM image and the corresponding dimensions of the two-tier microspheres (PS-coPAA $@ M S(T))$ in Figure S5, the height of the nanogaps among nanoparticles was approximately $100 \mathrm{~nm}$ and the minimum gap distance between two nanoparticles was about $15 \pm 10 \mathrm{~nm}$.

The surface of closely packed three-tier hierarchical microspheres of $f$-PS@MS exhibited a "rose petal” state, as presented in Figures $6 c$, with higher $C A$ and high adhesion to water. Hierarchical structures featured with micropapillae and nanofolds are known to exist on the petal surfaces of red roses. ${ }^{66}$ The produced film fabricated by three-tier microspheres was similar to the "rose petal" surface, where the three-tier microsphere units serve as the micropapillae, and nanowrinkles on the microsphere are like nanofolds on every micropapillae. Compared to the surface of assembled two-tier microspheres in Figure $6 \mathrm{~b}$, the three-tier microsphere assembled surface showed higher water $C A$ of $145^{\circ}$ as well as high adhesion to water. This is mainly attributed to the enhanced roughness and spatial frequency from the multiple-tier of nanostructures and microstructures. Figure $6 \mathrm{~d}$ shows that a water drop stably remained on the surface of assembled three-tier $f$-PS@MS microspheres (Figure 6c), even when it is turned $180^{\circ}$ upsidedown (Video S1).

Overall, these three states of surface wettability are mainly determined by the physical micro- and nanofeatured structures. In this way, by using the microfluidic platform, we could create the microunits with controllable nanostructures for constructing surfaces with variable wettability. With the combination of nanoparticles and polymeric materials, the multitier nano- and microstructures could be manipulated. Further modification of the film surfaces with tunable chemical compositions is expected to be able to better regulate the surface wettability in a better controllable manner.

\section{CONCLUSION}

In summary, we report a robust and facile method for the rapid fabrication of three-tier hierarchical microspheres featuring nanostructures using a droplet-based microfluidic platform, which has the advantages of precise control over particle size, flexible tunability of materials, and easy integration with other techniques. These hierarchical microspheres with multiple structures could be applied as constructable units to build up bioinspired surfaces by self-assembly on various substrates. Both the microsphere size and generation frequency can be controlled by the microfluidic device design and flow dynamics of oil and water phases. The nanometer-scale patterns on the microsphere stem from the self-assembly of colloidal nanoparticles. The nanowrinkles with sizes in the range of tens of nanometers are generated from the gradient polymerization-induced stress mismatch between the soft NIPAM monomers/oligomers that are strongly bound to PS surface via hydrogen bonds and the PNIPAM (polymerized NIPAM) hard skin layer. The roughness and spatial frequency of the nanowrinkles could be tailored by the density of carboxylic groups at the PS nanoparticle surfaces. Due to the morphology in three spatial levels at the nano- and microscale, these microspheres show high roughness and spatial frequency. Surfaces made from self-assembly of the two- and three-tier microspheres exhibit "gecko" and "rose petal" wetting states, showing hydrophobicity with CAs of 130 and $145^{\circ}$, respectively, but high water adhesion to water by holding a drop without falling off when the film is tilted. With implementation of chemical control over the microspheresnanoparticles-nanowrinkles, more applications could be expected for this reported method for creating 3D three-tier hierarchical structures as catalytic support, gas sensing units, and sample preconcentration materials.

\section{METHODS}

Materials. $N$-isopropylacrylamide (NIPAM, purity $\geq 99 \%$ ), $N, N$ methylene bis(acrylamide) (MBA, purity $\geq 99 \%$ ), $n$-hexadecane (purity $99 \%$ ), sorbitane monooleate sorbitan oleate (Span 80), and 2,2diethoxyacetophenone (DEAP, purity $\geq 98 \%$ were purchased from Sigma-Aldrich (The Netherlands). Both bare polystyrene nanoparticles (bare-PS nanoparticles, $d=250 \pm 10 \mathrm{~nm}$ ) and copolymerized polystyrene nanoparticles with two different acrylic acid (AA) molar fractions (10\% PS-co-PAA ${ }^{l}, d=250 \pm 10 \mathrm{~nm}$ nanoparticles, $18.5 \%$ PSco-PAA ${ }^{h}, d=250 \pm 10 \mathrm{~nm}$ ) were provided by Nanjing Nanorainbow Biotechnology Co., Ltd. company (Nanjing, China). The PS-co-PAA nanoparticles were synthesized from random polymerization by the monomer of styrene and acrylic acid with different contents via an soapfree emulsion polymerization method. The chemical structure of these different polystyrene nanoparticles used in this work is shown in Figure S3a. The fluorescent polystyrene nanoparticles with carboxylic functionalized surface (defined as $f$-PS nanoparticles, $d=250 \pm 50$ $\mathrm{nm}$ ) were purchased from Wuhan Jiayuan Quantumdots Co. Ltd. company (Wuhan, China). The synthetic route and chemical structure of $f$-PS nanoparticles are shown in Figure S3b. Hydrazine-modified polystyrene nanoparticles were treated further with the succinic anhydride, yielding the carboxylic-functionalized $f$-PS nanoparticles. ${ }^{67}$ The HR-SEM images of the morphological surface of bare-PS, PS-coPAA, and $f$-PS nanoparticles are shown in Figures $S 3 c-f$. Polystyrene nanoparticles were all dispersed in deionized water without any additions before using to generate emulsion droplets.

Fabrication of the Microfluidic Device. The polydimethylsiloxane (PDMS) microfluidic device with flow-focusing geometry was fabricated using the standard soft lithography technique. ${ }^{88}$ The detailed fabrication process of this microfluidic device was presented in our previous work. $^{69}$

Construction of Hierarchical Microspheres. Two immiscible phases were used to produce the highly uniform emulsion droplets through the flow-focusing microfluidic device. The water phase (dispersed phase) was a suspension of different polystyrene nanoparticles with a certain amount of monomer (NIPAM) and cross-linker (MBA). The oil phase (continuous phase) was $n$-hexadecane contained Span 80 (as the surfactant to stabilize the generated emulsion droplets) and DEAP (as the photoinitiator). The water phase was sheared and broken into monodispersed emulsion droplets by the oil phase in the flow focusing area of the microfluidic device. Produced monodispersed droplets containing numbers of nanoparticles served as a template to induce the self-assembly of nanoparticles on the droplet surface driven by surface energy minimization and stabilized by the electrostatic repulsion. As the time evolved, the droplets were featured with close- 
packed nanopatterns, which were then solidified upon UV irradiation (IntelliRy-600, $365 \mathrm{~nm}, 600 \mathrm{~W}, 120 \mathrm{~mW} \mathrm{~cm} \mathrm{c}^{-2}$, Uvitron International, Inc., Massachusetts) for $300 \mathrm{~s}$ through photopolymerization, yielding solid hierarchical microspheres (denoted as MS). In addition, the thermal evaporation method was also used to yield solid microspheres (denoted as MS $(T)$ ) as a control approach. Herein, the composition of hierarchical microcapsules can be precisely and flexibly tuned, which are attributed to the advantages of microfluidic technique.

Characterization. Optical Microscope. The generation process of emulsion droplets was monitored and recorded by using an inverted optical microscope (Olympus IX2, Tokyo, Japan) equipped with a high-speed camera (Phantom, MIRO MIIO, Vision Research Inc., Wayne County, NC, USA) in bright-field mode. The fluorescent images were taken by an inverted optical microscope (Olympus IX73, Tokyo, Japan) equipped with a digital camera (Olympus DP73, Tokyo, Japan) using the $365 \mathrm{~nm}$ excitation mode.

Scanning Electron Microscope. Fabricated hierarchical microsphere suspension in ethanol was dripped onto a clean piece of silicon substrate and dried naturally at room temperature. A thin platinum layer was coated on the exposed surface using a coating machine (Quorus, Q150T ES, Quorum Technologies Ltd., Ashford, England) prior to the HR-SEM examination. The morphology of these microspheres was characterized by FE-SEM (ZEISS ultra 55, Carl Zeiss, Germany) using either the secondary electron signal or in-lens signal with an accelerating voltage of $5 \mathrm{kV}$. The focus ion beam (FEI NOVA600 DUALBEAM FIB/SEM, United States) was used to cut the cross-section of wrinkled microspheres in order to characterize the structure and texture of $f$-PS nanoparticles encapsulated inside the microspheres.

Atomic Force Microscope. Dimensions of nanopatterns on the surface of two-tier/three-tier hierarchical microspheres were measured by using an atomic force microscope (AFM) (Bruker Fast Scan, Bruker Corporation, Billerica, United States) equipped with a Fast Scan scanner for high-resolution images. The contact-mode AFM with a tip radius of $2 \mathrm{~nm}$ and scanning frequency in the range of $0.99-1.99 \mathrm{~Hz}$ was used to collect the topological surface.

Fourier-Transform Infrared Spectroscopy. Polystyrene nanoparticles (PS-co-PAA and $f$-PS nanoparticles) were analyzed by using a Fourier-transform infrared spectroscopy (FT-IR) under the transmittance mode (PerkinElmer Spectrum 2000, USA) in the region of $400-4000 \mathrm{~cm}^{-1}$. Before each measurement, the sample was vacuumed and dried in an oven overnight. The dried samples were mixed with $\mathrm{KBr}$ by grinding and compressed into a round transparent sheet for FT-IR analysis. The adsorption spectra of PS-co-PAA nanoparticles, $f$-PS nanoparticles, and hierarchical microspheres ( $f$-PS@MS) made from $f$ PS nanoparticles analyzed by FI-TR are shown in Figure S6a1-c1, respectively. Figure S6a2-c2 shows the locally amplified transmittance spectra in the wavenumber of $1500-1800 \mathrm{~cm}^{-1}$ corresponding to Figure $S 6 a 1-c 1$.

The transmittance peaks at $3068,3026,1596,1492,1448,756$, and $696 \mathrm{~cm}^{-1}$ correspond to the phenyl $(\mathrm{Ph})$ group; the peaks at 2920 and $2846 \mathrm{~cm}^{-1}$ correspond to the methylene and methenyl groups, respectively; and the peaks at 1072 and $1024 \mathrm{~cm}^{-1}$ correspond to the $\mathrm{C}-\mathrm{C}$ and $\mathrm{C}-\mathrm{Ph}$ bonds, respectively (see spectra in Figure S6a1-c1). This indicated the presence of the bone polymer (polystyrene). Moreover, each spectrum showed its own characteristic peaks, demonstrating different functional groups produced on the corresponding particles. As shown in Figure S6a1-a2, the peaks located at 3448 and $1701 \mathrm{~cm}^{-1}$ were resulted from stretching vibrations of hydroxyl group and carbonyl group $\mathrm{C}=\mathrm{O}$, respectively, ${ }^{70}$ which indicated the existence of carboxylic group on PS-co-PAA nanoparticles. Figure S6b1 shows the presence of amide group and carboxylic group on the specifically carboxylic functionalized nanoparticles ( $f$-PS), resulting from the $\mathrm{N}-\mathrm{H}$ stretch at $3431 \mathrm{~cm}^{-1}$, the $\mathrm{C}=\mathrm{O}$ stretch from amide group at $1641 \mathrm{~cm}^{-1}$, and the weak peak of $\mathrm{O}-\mathrm{H}$ stretch and $\mathrm{C}=\mathrm{O}$ stretch from carboxylic group located at 3730 and $1745 \mathrm{~cm}^{-1}$, respectively. The produced three-tier hierarchical microspheres $(f-$ PS@MS) with nanowrinkles were also analyzed by using FT-IR (see Figure S6c1). The broad band at $3464 \mathrm{~cm}^{-1}$ is attributed to the $\mathrm{N}-\mathrm{H}$ stretching vibration, and the strong peak at $1647 \mathrm{~cm}^{-1}$ is due to $\mathrm{C}=\mathrm{O}$ stretching, demonstrating all typical absorption peaks of NIPAM polymer. ${ }^{91}$ The peak located at $1101 \mathrm{~cm}^{-1}$ indicated the stretch of the $\mathrm{C}-\mathrm{N}$ group.

$X$-ray Photoelectron Spectroscopy. X-ray photoelectron spectroscopy (XPS) was utilized for surface analysis, by using an Omicron Nanotechnology $\mathrm{GmbH}$ (Oxford Instruments) with a photon energy of $1486.7 \mathrm{eV}$ ( $\mathrm{Al} \mathrm{K} \alpha \mathrm{X}$-ray source) at a scanning step size of $0.1 \mathrm{eV}$. Before the measurement, the samples were dried and transferred onto a clean piece of carbon film. XPS spectra were obtained with a spot size of 200 $\mu \mathrm{m}$. High-resolution scans of individual elements and the survey spectra were measured at a pass energy of 20 and $200 \mathrm{eV}$, respectively. The scans were averaged over 10 sweeps.

The XPS spectra of the PS-co-PAA and $f$-PS nanoparticles are shown in Figure S7a,d, respectively. Obviously, the PS-co-PAA nanoparticles were composed of $\mathrm{C}$ and $\mathrm{O}$ elements without $\mathrm{N}$ element. The spectrum of $f$-PS nanoparticles showed the presence of $\mathrm{N}$ element, which is consistent with the chemical structure and component of the asprepared $f$-PSnanoparticles, as shown in Figure S3. The high-resolution spectrum of $\mathrm{C} 1 \mathrm{~s}$ from the PS-co-PAA nanoparticles (Figure $\mathrm{S} 7 \mathrm{~b}$ ) showed three peaks at 285.2, 286.7, and $289.2 \mathrm{eV}$ for $\mathrm{C}-\mathrm{C}, \mathrm{C}-\mathrm{O}$, and $\mathrm{C}(\mathrm{O}) \mathrm{O}$, respectively. The high-resolution spectrum of $\mathrm{O} 1 \mathrm{~s}$ (Figure $\mathrm{S} 7 \mathrm{c})$ revealed the presence of $\mathrm{C}-\mathrm{O}$ bonds $(532.5 \mathrm{eV})$ and $\mathrm{C}=\mathrm{O}$ bonds $(533.7 \mathrm{eV})$. Moreover, the high-resolution spectrum of $\mathrm{C} 1 \mathrm{~s}$ from $f$-PS nanoparticles (Figure S7e) showed three bands located at $\mathrm{C}-\mathrm{C}$ bonds $(284.0 \mathrm{eV}), \mathrm{C}-\mathrm{N}$ bonds $(285.7 \mathrm{eV})$, and $\mathrm{C}=\mathrm{O}(287.9 \mathrm{eV})$, respectively. The peaks at $\mathrm{C}-\mathrm{O}(531.1 \mathrm{eV})$ and $\mathrm{C}=\mathrm{O}(532.7 \mathrm{eV})$ were also presented in the high-resolution spectrum of $\mathrm{O} 1 \mathrm{~s}$ from $f$-PS nanoparticles (Figure S7f).

Preparation of Bioinspired Surfaces. The hierarchical microsphere suspension (dispersed in deionized water) was pipetted onto a piece of silicon wafer cleaned by using oxygen plasma. Due to hydrophobicity, the microspheres floated on the water surface and started to assemble at room temperature naturally as water evaporated. The obtained films with multilayer patterned microspheres were then used to study the wetting performance (Figure S8).

Contact Angle Measurement. One water drop with a volume of 2 $\mu \mathrm{L}$ was dripped gently on the as-prepared substrate. The substrate was covered with a layer of produced hierarchical microspheres. An interfacial tension meter OCA 15 Pro (Dataphysics, Germany) was used to measure the contact angle at ambient temperature. The Laplace-Young fitting method was used to measure the value of the contact angle. Every substrate was measured for five times at different positions to obtain the average contact angle.

\section{ASSOCIATED CONTENT}

\section{Supporting Information}

The Supporting Information is available free of charge on the ACS Publications website at DOI: 10.1021/acsnano.9b00245.

Estimation of the volume shrinkage fraction of droplets, the surface-to-volume ratio of a f-PS@MS, and the density of nanogaps, the schematic of different PS nanoparticles and the corresponding HR-SEM images, HR-SEM images of hierarchical microspheres made from PS-co-PAA ${ }^{l}$ nanoparticles, and PS-co-PAA ${ }^{h}$ nanoparticles, and AFM images of PS-co-PAA $@$ MS $(T)$, and FT-IR spectra, XPS spectra (PDF)

Video S1: Demonstration of one $2 \mu \mathrm{L}$ water drop sitting on the surface made from assembled three-tier hierarchical microspheres (MPG)

\section{AUTHOR INFORMATION}

\section{Corresponding Author}

*E-mail: shuill@m.scnu.edu.cn.

ORCID

Hai Le-The: 0000-0002-3153-2937

Zuankai Wang: 0000-0002-3510-1122 
Guofu Zhou: 0000-0003-1101-1947

Lingling Shui: 0000-0001-8517-1535

\section{Present Address}

${ }^{\perp}$ National Center for International Research on Green Optoelectronics and South China Academy of Advanced Optoelectronics, South China Normal University, Guangzhou 510006, China

\section{Notes}

The authors declare no competing financial interest.

\section{ACKNOWLEDGMENTS}

We appreciate the financial support from the National Natural Science Foundation of China (no. 61574065), Guangdong Science and Technology Planning Project (no. 2016B090906004), and Special Fund Project of Science and Technology Application (no. 2017B020240002), Pioneers in Healthcare voucher (project Ischemia on chip) of the University of Twente, MST and ZGT in The Netherlands, and the Oversea study of Guangzhou Elite Project support, China. The platforms of PCSIRT project no. IRT 17R40, the National 111 Project, and the MOE International Laboratory for Optical Information Technologies are also appreciated for providing technical support.

\section{REFERENCES}

(1) Visaveliya, N. R.; Leishman, C. W.; Ng, K.; Yehya, N.; Tobar, N.; Eisele, D. M.; Köhler, J. M. Structured Particulate Materials: Surface Wrinkling and Porosity of Polymer Particles toward Biological and Biomedical Applications. Adv. Mater. Interfaces 2017, 4, 17701251700929.

(2) Ryu, S. Y.; Seo, J. H.; Hafeez, H.; Song, M.; Shin, J. Y.; Kim, D. H.; Jung, Y. C.; Kim, C. S. Effects of the Wrinkle Structure and Flat Structure Formed during Static Low-Temperature Annealing of $\mathrm{ZnO}$ on the Performance of Inverted Polymer Solar Cells. J. Phys. Chem. C 2017, 121, 9191-9201.

(3) Liu, S.; Deng, R.; Li, W.; Zhu, J. Polymer Microparticles with Controllable Surface Textures Generated through Interfacial Instabilities of Emulsion Droplets. Adv. Funct. Mater. 2012, 22, 1692-1697.

(4) Lei, Y.; Sun, R.; Zhang, X.; Feng, X.; Jiang, L. Oxygen Rich Enzyme Biosensor Based on Superhydrophobic Electrode. Adv. Mater. 2016, 28, 1477-1481.

(5) Chung, J. Y.; Youngblood, J. P.; Stafford, C. M. Anisotropic Wetting on Tunable Micro-Wrinkled Surfaces. Soft Matter 2007, 3, $1163-1169$

(6) Khare, K.; Zhou, J.; Yang, S. Tunable Open-Channel Microfluidics on Soft Poly (dimethylsiloxane)(PDMS) Substrates with Sinusoidal Grooves. Langmuir 2009, 25, 12794-12799.

(7) Ohzono, T.; Monobe, H. Morphological Transformation of a Liquid Micropattern on Dynamically Tunable Microwrinkles. Langmuir 2010, 26, 6127-6132.

(8) Parihar, V.; Bandyopadhyay, S.; Das, S.; Dasgupta, S. Anisotropic Electrowetting on Wrinkled Surfaces: Enhanced Wetting and Dependency on Initial Wetting State. Langmuir 2018, 34, 1844-1854.

(9) Efimenko, K.; Rackaitis, M.; Manias, E.; Vaziri, A.; Mahadevan, L.; Genzer, J. Nested Self-Similar Wrinkling Patterns in Skins. Nat. Mater. 2005, 4, 293-297.

(10) Zhang, L.; Lang, X.; Hirata, A.; Chen, M. Wrinkled Nanoporous Gold Films with Ultrahigh Surface-Enhanced Raman Scattering Enhancement. ACS Nano 2011, 5, 4407-4413.

(11) Visaveliya, N.; Lenke, S.; Koehler, J. M. Composite Sensor Particles for Tuned SERS Sensing: Microfluidic Synthesis, Properties and Applications. ACS Appl. Mater. Interfaces 2015, 7, 10742-10754.

(12) Bae, H. J.; Bae, S.; Park, C.; Han, S.; Kim, J.; Kim, L. N.; Kim, K.; Song, S. H.; Park, W.; Kwon, S. Biomimetic Microfingerprints for Anti Counterfeiting Strategies. Adv. Mater. 2015, 27, 2083-2089.
(13) Xie, M.; Xu, F.; Zhang, L.; Yin, J.; Jiang, X. Reversible Surface Dual-Pattern with Simultaneously Dynamic Wrinkled Topography and Fluorescence. ACS Macro Lett. 2018, 7, 540-545.

(14) Kim, J. B.; Kim, P.; Pégard, N. C.; Oh, S. J.; Kagan, C. R.; Fleischer, J. W.; Stone, H. A.; Loo, Y.-L. Wrinkles and Deep Folds as Photonic Structures in Photovoltaics. Nat. Photonics 2012, 6, 327-332.

(15) Kim, D.-H.; Ahn, J.-H.; Choi, W. M.; Kim, H.-S.; Kim, T.-H.; Song, J.; Huang, Y. Y.; Liu, Z.; Lu, C.; Rogers, J. A. Stretchable and Foldable Silicon Integrated Circuits. Science 2008, 320, 507-511.

(16) Khang, D.-Y.; Jiang, H.; Huang, Y.; Rogers, J. A. A Stretchable Form of Single-Crystal Silicon for High-Performance Electronics on Rubber Substrates. Science 2006, 311, 208-212.

(17) Harrison, C.; Stafford, C. M.; Zhang, W.; Karim, A. Sinusoidal Phase Grating Created by a Tunably Buckled Surface. Appl. Phys. Lett. 2004, 85, 4016-4018.

(18) Hano, N.; Takafuji, M.; Ihara, H. One-Pot Preparation of Polymer Microspheres Having Wrinkled Hard Surfaces through SelfAssembly of Silica Nanoparticles. Chem. Commun. 2017, 53, 91479150.

(19) Dong, H.; Tang, G.; Ma, T.; Cao, X. One-Step Fabrication of Inorganic/Organic Hybrid Microspheres with Tunable Surface Texture for Controlled Drug Release Application. J. Mater. Sci.: Mater. Med. 2016, $27,7$.

(20) del Campo, A.; Arzt, E. Fabrication Approaches for Generating Complex Micro- and Nanopatterns on Polymeric Surfaces. Chem. Rev. 2008, 108, 911-945.

(21) Martina, M.; Subramanyam, G.; Weaver, J. C.; Hutmacher, D. W.; Morse, D. E.; Valiyaveettil, S. Developing Macroporous Bicontinuous Materials as Scaffolds for Tissue Engineering. Biomaterials 2005, 26, 5609-5616.

(22) Ryu, J.; Kim, W.; Yun, J.; Lee, K.; Lee, J.; Yu, H.; Kim, J. H.; Kim, J. J.; Jang, J. Fabrication of Uniform Wrinkled Silica Nanoparticles and Their Application to Abrasives in Chemical Mechanical Planarization. ACS Appl. Mater. Interfaces 2018, 10, 11843-11851.

(23) Efimenko, K.; Finlay, J.; Callow, M. E.; Callow, J. A.; Genzer, J. Development and Testing of Hierarchically Wrinkled Coatings for Marine Antifouling. ACS Appl. Mater. Interfaces 2009, 1, 1031-1040.

(24) Chen, C. M.; Yang, S. Wrinkling Instabilities in Polymer Films and Their Applications. Polym. Int. 2012, 61, 1041-1047.

(25) Uchida, N.; Ohzono, T. Orientational Ordering of BucklingInduced Microwrinkles on Soft Substrates. Soft Matter 2010, 6, 57295735.

(26) Yang, S.; Khare, K.; Lin, P. C. Harnessing Surface Wrinkle Patterns in Soft Matter. Adv. Funct. Mater. 2010, 20, 2550-2564.

(27) San Lim, H.; Lee, S. Y.; Lee, N. E.; Cho, S. O. Patterning of Wrinkled Polymer Surfaces by Single-Step Electron Irradiation. Langmuir 2018, 34, 5290-5296.

(28) Bowden, N.; Brittain, S.; Evans, A. G.; Hutchinson, J. W.; Whitesides, G. M. Spontaneous Formation of Ordered Structures in Thin Films of Metals Supported on an Elastomeric Polymer. Nature 1998, 393, 146-149.

(29) Kim, H. S.; Crosby, A. J. Solvent Responsive Surface via Wrinkling Instability. Adv. Mater. 2011, 23, 4188-4192.

(30) Chua, D. B.; Ng, H.; Li, S. F. Spontaneous Formation of Complex and Ordered Structures on Oxygen-Plasma-Treated Elastomeric Polydimethylsiloxane. Appl. Phys. Lett. 2000, 76, 721-723.

(31) Liu, Y.; Moevius, L.; Xu, X.; Qian, T.; Yeomans, J. M.; Wang, Z. Pancake Bouncing on Superhydrophobic Surfaces. Nat. Phys. 2014, 10, 515-519.

(32) Lai, Y.; Gao, X.; Zhuang, H.; Huang, J.; Lin, C.; Jiang, L. Designing Superhydrophobic Porous Nanostructures with Tunable Water Adhesion. Adv. Mater. 2009, 21, 3799-3803.

(33) Zhang, M.; Wang, P.; Sun, H.; Wang, Z. Superhydrophobic Surface with Hierarchical Architecture and Bimetallic Composition for Enhanced Antibacterial Activity. ACS Appl. Mater. Interfaces 2014, 6, 22108-22115.

(34) Guo, P.; Zheng, Y.; Wen, M.; Song, C.; Lin, Y.; Jiang, L. Icephobic/Anti Icing Properties of Micro/Nanostructured Surfaces. Adv. Mater. 2012, 24, 2642-2648. 
(35) Li, J.; Liang, G.; Zhu, X.; Yang, S. Exploiting Nanoroughness on Holographically Patterned Three Dimensional Photonic Crystals. Adv. Funct. Mater. 2012, 22, 2980-2986.

(36) Shiu, J.-Y.; Kuo, C.-W.; Chen, P.; Mou, C.-Y. Fabrication of Tunable Superhydrophobic Surfaces by Nanosphere Lithography. Chem. Mater. 2004, 16, 561-564.

(37) Liu, W.; Liu, X.; Fangteng, J.; Wang, S.; Fang, L.; Shen, H.; Xiang, S.; Sun, H.; Yang, B. Bioinspired Polyethylene Terephthalate Nanocone Arrays with Underwater Superoleophobicity and Anti-Bioadhesion Properties. Nanoscale 2014, 6, 13845-13853.

(38) Gao, X.; Yan, X.; Yao, X.; Xu, L.; Zhang, K.; Zhang, J.; Yang, B.; Jiang, L. The Dry Style Antifogging Properties of Mosquito Compound Eyes and Artificial Analogues Prepared by Soft Lithography. Adv. Mater. 2007, 19, 2213-2217.

(39) Li, M.; Joung, D.; Hughes, B.; Waldman, S. D.; Kozinski, J. A.; Hwang, D. K. Wrinkling Non-Spherical Particles and Its Application in Cell Attachment Promotion. Sci. Rep. 2016, 6, 30463.

(40) Bock, N.; Woodruff, M. A.; Hutmacher, D. W.; Dargaville, T. R. Electrospraying, a Reproducible Method for Production of Polymeric Microspheres for Biomedical Applications. Polymers 201 1, 3, 131-149.

(41) Zhang, M.-J.; Wang, W.; Yang, X.-L.; Ma, B.; Liu, Y.-M.; Xie, R.; Ju, X.-J.; Liu, Z.; Chu, L.-Y. Uniform Microparticles with Controllable Highly Interconnected Hierarchical Porous Structures. ACS Appl. Mater. Interfaces 2015, 7, 13758-13767.

(42) Serra, C. A.; Chang, Z. Microfluidic Assisted Synthesis of Polymer Particles. Chem. Eng. Technol. 2008, 31, 1099-1115.

(43) Ohm, C.; Serra, C.; Zentel, R. A Continuous Flow Synthesis of Micrometer Sized Actuators from Liquid Crystalline Elastomers. Adv. Mater. 2009, 21, 4859-4862.

(44) Stafford, C. M.; Harrison, C.; Beers, K. L.; Karim, A.; Amis, E. J.; VanLandingham, M. R.; Kim, H.-C.; Volksen, W.; Miller, R. D.; Simonyi, E. E. A Buckling-Based Metrology for Measuring the Elastic Moduli of Polymeric Thin Films. Nat. Mater. 2004, 3, 545-550.

(45) Lin, P.-C.; Yang, S. Spontaneous Formation of One-Dimensional Ripples in Transit to Highly Ordered Two-Dimensional Herringbone Structures through Sequential and Unequal Biaxial Mechanical Stretching. Appl. Phys. Lett. 2007, 90, 241903.

(46) Yin, J.; Han, X.; Cao, Y.; Lu, C. Surface Wrinkling on Polydimethylsiloxane Microspheres via Wet Surface Chemical Oxidation. Sci. Rep. 2015, 4, 5710.

(47) Trindade, A.; Canejo, J. P.; Pinto, L.; Patrício, P.; Brogueira, P.; Teixeira, P. I. C.; Godinho, M. Wrinkling Labyrinth Patterns on Elastomeric Janus Particles. Macromolecules 2011, 44, 2220-2228.

(48) Im, J.; Yoo, D.; Kim, J.; Yoon, S.; Cho, K. Y. One Step Spontaneous Formation of Dual Wrinkling on Uniform Sized Microparticles Induced by Surface. Macromol. Chem. Phys. 2017, 218, 1700152

(49) Chandra, D.; Crosby, A. J. Self Wrinkling of UV Cured Polymer Films. Adv. Mater. 2011, 23, 3441-3445.

(50) Wang, J.; Eijkel, J. C.; Jin, M.; Xie, S.; Yuan, D.; Zhou, G.; van den Berg, A.; Shui, L. Microfluidic Fabrication of Responsive Hierarchical Microscale Particles from Macroscale Materials and Nanoscale Particles. Sens. Actuators, B 2017, 247, 78-91.

(51) Zhao, X.; Cao, Y.; Ito, F.; Chen, H. H.; Nagai, K.; Zhao, Y. H.; $\mathrm{Gu}, \mathrm{Z}$. Z. Colloidal Crystal Beads as Supports for Biomolecular Screening. Angew. Chem., Int. Ed. 2006, 45, 6835-6838.

(52) Tamai, Y.; Tanaka, H.; Nakanishi, K. Molecular Dynamics Study of Polymer-Water Interaction in Hydrogels. 1. Hydrogen-Bond Structure. Macromolecules 1996, 29, 6750-6760.

(53) Wang, J.; Lin, L.; Cheng, Q.; Jiang, L. A Strong Bio Inspired Layered PNIPAM-Clay Nanocomposite Hydrogel. Angew. Chem., Int. Ed. 2012, 51, 4676-4680.

(54) Basu, S. K.; Scriven, L.; Francis, L.; McCormick, A. Mechanism of Wrinkle Formation in Curing Coatings. Prog. Org. Coat. 2005, 53, 116.

(55) Sun, Q.; Deng, Y. In situ Synthesis of Temperature-Sensitive Hollow Microspheres via Interfacial Polymerization. J. Am. Chem. Soc. 2005, 127, 8274-8275.
(56) Ono, Y.; Shikata, T. Hydration and Dynamic Behavior of Poly (N-isopropylacrylamide)s in Aqueous Solution: A Sharp Phase Transition at the Lower Critical Solution Temperature. J. Am. Chem. Soc. 2006, 128, 10030-10031.

(57) Schmidt, S.; Zeiser, M.; Hellweg, T.; Duschl, C.; Fery, A.; Möhwald, H. Adhesion and Mechanical Properties of PNIPAM Microgel Films and Their Potential Use as Switchable Cell Culture Substrates. Adv. Funct. Mater. 2010, 20, 3235-3243.

(58) Groenewold, J. Wrinkling of Plates Coupled with Soft Elastic Media. Phys. A 2001, 298, 32-45.

(59) Lee, J.-H.; Ro, H. W.; Huang, R.; Lemaillet, P.; Germer, T. A.; Soles, C. L.; Stafford, C. M. Anisotropic, Hierarchical Surface Patterns via Surface Wrinkling of Nanopatterned Polymer Films. Nano Lett. 2012, 12, 5995-5999.

(60) Wen, M.; Wang, L.; Zhang, M.; Jiang, L.; Zheng, Y. Antifogging and Icing-Delay Properties of Composite Micro- and Nanostructured Surfaces. ACS Appl. Mater. Interfaces 2014, 6, 3963-3968.

(61) Zhang, M.; Wang, L.; Feng, S.; Zheng, Y. A Strategy of Antifogging: Air-Trapped Hollow Microsphere Nanocomposites. Chem. Mater. 2017, 29, 2899-2905.

(62) Faustini, M.; Nicole, L.; Boissiere, C.; Innocenzi, P.; Sanchez, C.; Grosso, D. Hydrophobic, Antireflective, Self-Cleaning, and Antifogging Sol-Gel Coatings: An Example of Multifunctional Nanostructured Materials for Photovoltaic Cells. Chem. Mater. 2010, 22, 4406-4413.

(63) Wenzel, R. N. Resistance of Solid Surfaces to Wetting by Water. Ind. Eng. Chem. 1936, 28, 988-994.

(64) Cassie, A.; Baxter, S. Wettability of Porous Surfaces. Trans. Faraday Soc. 1944, 40, 546-551.

(65) Jin, M.; Feng, X.; Feng, L.; Sun, T.; Zhai, J.; Li, T.; Jiang, L. Superhydrophobic Aligned Polystyrene Nanotube Films with High Adhesive Force. Adv. Mater. 2005, 17, 1977-1981.

(66) Feng, L.; Zhang, Y.; Xi, J.; Zhu, Y.; Wang, N.; Xia, F.; Jiang, L. Petal Effect: A Superhydrophobic State with High Adhesive Force. Langmuir 2008, 24, 4114-4119.

(67) Qhobosheane, M.; Santra, S.; Zhang, P.; Tan, W. Biochemically Functionalized Silica Nanoparticles. Analyst 2001, 126, 1274-1278.

(68) Xia, Y.; Whitesides, G. M. Soft Lithography. Angew. Chem., Int. Ed. 1998, 37, 550-575.

(69) Wang, J.; Jin, M.; Gong, Y.; Li, H.; Wu, S.; Zhang, Z.; Zhou, G.; Shui, L.; Eijkel, J. C.; van den Berg, A. Continuous Fabrication of Microcapsules with Controllable Metal Covered Nanoparticle Arrays Using Droplet Microfluidics for Localized Surface Plasmon Resonance. Lab Chip 2017, 17, 1970-1979.

(70) Hojjati, B.; Sui, R.; Charpentier, P. A. Synthesis of $\mathrm{TiO}_{2} / \mathrm{PAA}$ Nanocomposite by RAFT Polymerization. Polymer 2007, 48, 58505858.

(71) Kundu, A.; Nandi, S.; Das, P.; Nandi, A. K. Fluorescent Graphene Oxide via Polymer Grafting: An Efficient Nanocarrier for both Hydrophilic and Hydrophobic Drugs. ACS Appl. Mater. Interfaces 2015, 7, 3512-3523. 